Pure and Applied Mathematics Quarterly

Volume 6, Number 2

(Special Issue: In honor of

Michael Atiyah and Isadore Singer)

307-329, 2010

\title{
Spectral Multiplicity and Odd K-theory
}

\author{
Ronald G. Douglas and Jerome Kaminker
}

\begin{abstract}
In this paper we begin a study of families of unbounded selfadjoint Fredholm operators with compact resolvant. The goal is to incorporate the information in the eigenspaces and eigenvalues of the operators, particularly the role that the multiplicity of eigenvalues plays, in obtaining topological invariants of the families.
\end{abstract}

Keywords: K-theory, unbounded selfadjoint Fredholms

\section{INTRODUCTION}

In the early sixties, K-theory, a generalized cohomology theory was defined by Atiyah and Hirzebruch, [2] based on a construction of Grothendieck used earlier in algebraic geometry. Following some spectacular applications in topology, the development of K-theory was intrinsically related the index theorem of Atiyah and Singer, [3]. One result of this entanglement was the realization, by Atiyah, [1] and Jänich, [8] of elements of the even group as homotopy classes of maps into the Fredholm operators. For the odd group, Atiyah and Singer showed in [4] that one could use homotopy classes of maps into the space of self-adjoint Fredholm operators.

Singer raised the question, [14], of describing elements in the cohomology of the space of self-adjoint Fredholm operators in a concrete way. The generator of

Received January 3, 2007.

1991 Mathematics Subject Classification. 19K56, 58J30, 46L87.

The research of the first author was supported in part by the National Science Foundation. 
the first cohomology group can be related to spectral flow and the eta invariant following the work of Atiyah-Patodi-Singer, [6], on the index formula for certain elliptic boundary value problems. This notion has proved pivotal in a number of directions, including physics, where it was used in the study of anomalies, [5]. Let us be a little more precise.

In [4] Atiyah and Singer established homotopy equivalences between various realizations of the odd K-theory group. The proofs involved, among other things, a careful analysis of finite portions of the spectrum of the operators. However, the precise relationships of some of these objects were left unresolved. In subsequent years, there has been some follow up on these ideas but analyzing families has turned to other notions such as gerbes which can involve ancillary structure. Our goal in this paper is to return to the framework introduced in [4] and attempt to relate the odd classes directly to the behavior of the self-adjoint operators.

One can show that spectral flow is determined by just a knowledge of the behavior of the eigenvalues of the family, along with their multiplicities. However, that is not true for the class in K-theory. To overcome this defect, one must bring in the behavior of the eigenspaces as well. Hence, we seek to unravel this dependence and understand how to obtain invariants similar to characteristic classes. We believe it is likely that these relationships will have applications to physics, such as "higher anomalies", and lead to a study of "higher" spectral flow and index theory in general. The authors would like to thank Ryszard Nest for hospitality during a visit to the University of Copenhagen where this project began. We would also like to thank Alan Carey who took part in initial discussions on this topic and provided valuable insights.

This paper should be viewed as a first step in which some basic structure is revealed and some results are obtained. One goal here is to formulate basic questions and frame critical issues which merit further investigation. Before providing an overview of our results we need to introduce some definitions and notation.

We begin with more details on the space of self-adjoint operators. It is well known that the space of bounded self-adjoint Fredholm operators on a separable Hilbert space $\mathcal{H}$, with the norm topology, is a classifying space for odd K-theory. That is, for any compact Hausdorff space, $X$, one has

$$
K^{1}(X) \cong\left[X, \mathcal{F}^{s a}\right]
$$


Here we are letting $\mathcal{F}^{s a}$ denote the component of the space of bounded self-adjoint Fredholm operators which have both positive and negative essential spectrum. We will also consider the subspace, $\mathcal{F}_{0}^{s a}$, consisting of operators, $T$, with $\|T\| \leq 1$ and the essential spectrum of $T$ equal to $\{ \pm 1\}$.

In applications one is often provided with a family of unbounded Fredholm operators parametrized by a locally path connected and connected space $X$. To be more precise we will consider the following subset of unbounded self-adjoint Fredholm operators.

Definition 1.1. The regular unbounded self-adjoint Fredholm operators, denoted $\mathcal{F}_{R}^{\text {sa }}$, consists of linear operators, $T$, which satisfy

i) $T$ is closed and self-adjoint,

ii) $\left(I+T^{2}\right)^{-1}$ is compact,

iii) $T$ has infinitely many positive and infinitely many negative eigenvalues.

Remark 1.1. We may also consider non-self-adjoint operators, $T$, satisfying the condition that $T^{*} T \in \mathcal{F}_{R}^{s a}$. Statements made about $\mathcal{F}_{R}^{s a}$ will hold with appropriate modifications for such operators which we will denote by $\mathcal{F}_{R}$.

We shall study families of operators,

$$
D=\left\{D_{x}\right\}: X \rightarrow \mathcal{F}_{R}^{s a} .
$$

Let $C_{b}(\mathbb{R})$ denote the bounded continuous functions on $\mathbb{R}$ for which the limits at $\pm \infty$ exist. We consider families which are continuous in the sense that the function

$$
\Theta: C_{b}(\mathbb{R}) \times X \rightarrow \mathcal{B}(\mathcal{H})
$$

defined by $\Theta(f, x)=f\left(D_{x}\right)$ is norm continuous. There is a topology on the set $\mathcal{F}_{R}^{s a}$ for which this holds.

With the Riesz topology, which is the one determined by the bounded transform, $D \mapsto D\left(I+D^{2}\right)^{-\frac{1}{2}}$, it has been shown by L. Nicolaescu in [13] that the space of unbounded self-adjoint Fredholms satisfying (i) and (iii) in Definition 1.1, but not necessarily (ii), provides a classifying space for odd K-theory. There is a related result by M. Joachim in [9] which states that those satisfying (ii) as well also form a classifying space. We will not need to make use of these results in the present paper, but they will be relevant for future work. 
The main examples will be families of Dirac operators on an odd-dimensional manifold $M$ parametrized by a compact space, $X$.

A family $\left\{D_{x}\right\}$, as above, determines an element of $K^{1}(X)$. It is obtained by applying the function $\chi(x)=x\left(1+x^{2}\right)^{-1 / 2}$ to each operator $D_{x}$ to obtain the family of bounded self-adjoint Fredholm operators

$$
\left\{\tilde{D}_{x}\right\}=\left\{\chi\left(D_{x}\right)\right\} .
$$

Then each operator in the resulting family $\left\{\tilde{D}_{x}\right\}$ is a bounded self-adjoint Fredholm operator and the homotopy class of the family yields an element of $K^{1}(X)$.

The Chern character of such a family, viewed in real cohomology, has components only in odd degrees,

$$
\operatorname{ch}\left(\left\{\chi\left(D_{x}\right)\right\}\right) \in \bigoplus_{i \geq 0} H^{2 i+1}(X, \mathbb{R}) .
$$

The class in $H^{1}(X, \mathbb{R})$ corresponds to spectral flow, and the class in $H^{3}(X, \mathbb{R})$ is determined by the index gerbe, c.f. [10]. One goal of the present work is to develop a method that leads to a different description of these classes and the higher dimensional classes which obstruct the triviality of the K-theory class associated to the family. These obstructions are to be determined explicitly in terms of the spectrum and eigenspaces of the operators in the family. This is in a spirit similar to spectral flow as we mentioned earlier. As a first step, in the present paper we will consider the role that the multiplicity of eigenvalues plays.

\section{The multiplicity of eigenvalues}

We will recall some basic definitions and facts that we will use.

Proposition 2.1. Let $D$ be an unbounded self-adjoint operator as above. Let $\lambda$ be an eigenvalue of $D$. Let $\delta>0$ be such that there is no other eigenvalue in $[\lambda-\delta, \lambda+\delta]$. Then the spectral projection onto the eigenspace for $\lambda$ is

$$
P_{\lambda}(D)=\frac{1}{2 \pi i} \int_{|z|=\delta} \frac{d z}{(z-D)} .
$$

and the multiplicity of $\lambda$ is given by $m(\lambda, D)=\operatorname{rank}\left(P_{\lambda}(D)\right)$.

Now, consider a family of operators, $\left\{D_{x}\right\}$. We introduce the following terminology. 
Definition 2.2. The graph (or spectral graph) of the family $\left\{D_{x}\right\}, \Gamma\left(\left\{D_{x}\right\}\right) \subseteq$ $X \times \mathbb{R}$, is

$$
\Gamma\left(\left\{D_{x}\right\}\right)=\left\{(y, \lambda) \mid \lambda \text { is an eigenvalue of } D_{y}\right\}
$$

Note that $\Gamma\left(\left\{D_{x}\right\}\right)$ is a closed subset of $X \times \mathbb{R}$. When the specific family is clear from the context, we will simply use $\Gamma$ and simply call it the graph, dropping the term "spectral".

Both the spectral projection and the multiplicity of eigenvalues define functions on the graph of the family. We must consider continuity properties of these functions.

Let $(x, \lambda) \in \Gamma$ be a point in the graph of the family.

Definition 2.3. A canonical neighborhood of $(x, \lambda)$ is one of the form $V \times(\lambda-$ $\delta, \lambda+\delta)$, where $x \in V, \delta>0$, such that

a) $\lambda$ is the only eigenvalue of $D_{x}$ in $(\lambda-\delta, \lambda+\delta)$,

b) if $k=m\left(D_{x}, \lambda\right)$, then, for each $y \in V$, one has

$$
\sum_{(y, \mu) \in(V \times(\lambda-\delta, \lambda+\delta)) \cap \Gamma} m\left(D_{y}, \mu\right)=k
$$

for each $y \in V$.

Proposition 2.4. Every point $(x, \lambda)$ in $\Gamma$ admits a canonical neighborhood, $V \times$ $(\lambda-\delta, \lambda+\delta)$ such that if $\lambda-\delta<\lambda_{1}(y) \leq \ldots \leq \lambda_{k}(y)<\lambda+\delta$ are the eigenvalues of $D_{y}$ in the given interval, then each $\lambda_{j}(y)$ is continuous on $V$.

Proof. This will follow from a corresponding statement for bounded operators in [7]. However, we will need a precise form of this fact so we recall the steps. Let $f(t)=t / \sqrt{1+t^{2}}-\lambda / \sqrt{1+\lambda^{2}}$, and consider the family of bounded operators $\left\{f\left(D_{x}\right)\right\}$. Choose a $\delta>0$ so that there is no other eigenvalue of $D_{x}$ in $(\lambda-\delta, \lambda+\delta)$. Assume $m\left(D_{x}, \lambda\right)=k$. Consider $f\left(D_{x}\right)$ and $f(\delta)$ and apply [7, p. 138], to obtain a neighborhood $V$ of $x$ such that for $y \in V$ there are exactly $k$ eigenvalues of $f\left(D_{y}\right)$ in $(f(\lambda-\delta), f(\lambda+\delta))$, which we will label

$$
-f(\delta)<\tilde{\lambda}_{0}(y) \leq \tilde{\lambda}_{1}(y) \leq \ldots \leq \tilde{\lambda}_{k}(y)<f(\delta),
$$

Moreover, $\left|\tilde{\lambda}_{j}(y)-\tilde{\lambda}_{j}\left(y^{\prime}\right)\right|<\left\|f\left(D_{y}\right)-f\left(D_{y^{\prime}}\right)\right\|$. Then $\lambda_{j}(y)=f^{-1}\left(\tilde{\lambda}_{j}(y)\right), V$, and $\delta$ yield the conclusion. 
As a corollary one obtains the following refinement.

Proposition 2.5. Let $\lambda_{0}(x)<\ldots<\lambda_{n}(x)$ be a list of the distinct eigenvalues of $\operatorname{spec}\left(D_{x}\right)$ which lie in a bounded interval of $\mathbb{R}$. Then there are disjoint canonical neighborhoods of each $\left(x, \lambda_{j}(x)\right)$, all with the same base $V$.

Proof. This follows easily from the method of proof of Proposition (2.4)

Note that there can be no points of the graph between the standard neighborhoods obtained.

It follows easily from this argument that the multiplicity function will be lower semi-continuous. The next result describes the conditions under which it is actually continuous at a point $(x, \lambda) \in \Gamma$.

Proposition 2.6. Let $U$ be a canonical neighborhood of $(x, \lambda) \in \Gamma$. The following are equivalent.

i) There is a positive integer $k$ so that the multiplicity function is constantly equal to $k$ on $U$,

ii) There is a $\delta>0$ and a neighborhood $V$ of $x$ so that, for each $y \in V, D_{y}$ has only one eigenvalue in the interval $[\lambda-\delta, \lambda+\delta]$,

iii) The function associating the spectral projection to a point in the graph is norm continuous on $\Gamma \cap(V \times[\lambda-\delta, \lambda+\delta])$.

Definition 2.7. The family $\left\{D_{x}\right\}$ has constant multiplicity $\mathrm{k}$ at $(x, \lambda)$ if it satisfies the conditions in Proposition 2.6.

We will next consider criteria for the triviality of the K-theory class associated to a family of self-adjoint operators.

Proposition 2.8. Let $\left\{D_{x}\right\}$ be a continuous family of self-adjoint operators. The following are equivalent.

i) The family defines the trivial element in $K^{1}(X)$,

ii) $\left\{D_{x}\right\}$ is homotopic to a family $\left\{D_{x}^{\prime}\right\}$ for which there is a continuous function, $\sigma: X \rightarrow \mathbb{R}$, such that $\sigma(x)$ is not an eigenvalue of $D_{x}^{\prime}$ for each $x$,

iii) $\left\{D_{x}\right\}$ is homotopic to a family $\left\{D_{x}^{\prime}\right\}$ for which there is a norm continuous family of projections $\left\{P_{x}^{\prime}\right\}$ with range the sum of the eigenspaces for positive eigenvalues. 
iv) There exists a spectral section for the family $\left\{D_{x}\right\}$, in the sense of MelrosePiazza, [11]. (i.e. there is a norm continuous family of projections which agree with the projections onto the positive eigenspaces outside of a closed interval, the interval itself depending continuously on $x$.)

Proof. This follows using the steps in the proof of Proposition 1 in Melrose-Piazza, [11].

\section{Spectral EXhaustions AND SPECTRAL FLOW}

In this section we will prove the existence and essential uniqueness of spectral exhaustions. Let $\left\{D_{x}\right\}$ be a continuous family of operators parametrized by the compact space $X$, which we assume for now is a simplicial complex.

Definition 3.1. A spectral exhaustion for the family $\left\{D_{x}\right\}$ is a family, of continuous functions $\mu_{n}: X \rightarrow \mathbb{R}$, indexed by $\mathbb{Z}$, satisfying

i) $\mu_{n}(x)$ is an eigenvalue of $D_{x}$ for each $x$,

ii) $\left\{\mu_{n}(x): n \in \mathbb{Z}\right\}$ exhausts the spectrum of $D_{x}$ counting multiplicity, for each $x$,

iii) for each $x$ and for each $n \in \mathbb{Z}, \mu_{n}(x) \leq \mu_{n+1}(x)$.

Remark 3.1. Note that, if the graphs of functions $\mu_{n}$ and $\mu_{n-1}$ are disjoint and the parameter space $X$ is connected, then $\mu_{n}(x)>\mu_{n-1}(x)$, for all $x$, so $\sigma(x)=\frac{1}{2}\left(\mu_{n}(x)-\mu_{n-1}(x)\right)$ satisfies condition (ii) of Proposition 2.8. Thus, the K-theory class of a family admitting a spectral exhaustion with this property is trivial.

Definition 3.2. An enumeration of the spectrum of an operator $D \in \mathcal{F}_{R}^{s a}$ is a function $e_{D}: \mathbb{Z} \rightarrow \mathbb{R}$ mapping $\mathbb{Z}$ onto the spectrum of $D$ and satisfying

i) if $\lambda$ is an eigenvalue of $D$ of multiplicity $k$, then there is an integer $N$ such that $\lambda=e_{D}(N)=e_{D}(N+1)=\ldots=e_{D}(N+k)$, and

ii) $e_{D}(n) \leq e_{D}(n+1)$ for all $n$.

Our goal in this section is to show that, if the spectral flow of the family $\left\{D_{x}\right\}$ is zero, one can construct an enumeration of the the spectrum of $D_{x}$, for each $x$, in such a way that the functions $\mu_{n}(x)=e_{D_{x}}(n)$ are continuous. Thus, we will obtain a spectral exhaustion for $\left\{D_{x}\right\}$. 
Proposition 3.3. Given an operator, D, an enumeration of the spectrum always exists and any two differ by translation by an integer.

Proof. Choose an eigenvalue, $\lambda$, of multiplicity $k$. We set $e_{D}(0)=\lambda$ and $e_{D}(-k+$ $1)=\ldots=e_{D}(0)=\lambda$. One can now uniquely extend this labeling to the rest of the spectrum. It is easy to check that this process provides an enumeration of the spectrum of $D$. Now suppose that $f_{D}$ is another one. We will show that there is an $N$ such that $f_{D}(n+N)=e_{D}(n)$ for all $n$. Let $\lambda$ be a point in the spectrum and let $n_{0}, m_{0}$ be the largest integers so that $e_{D}\left(n_{0}\right)=\lambda=f_{D}\left(m_{0}\right)$. Let $N=m_{0}-n_{0}$. Then it is easy to check that $e_{D}(n)=f_{D}(n+N)$ for all $n$.

Note that the existence of an integer $n$ such that $e_{D}(n)=f_{D}(n)$ is not sufficient to guarantee that $e_{D}=f_{D}$. However, if there is an integer $N$ such that $e_{D}(N)=$ $f_{D}(N)$ and $e_{D}(N+1)>e_{D}(N), f_{D}(N+1)>f_{D}(N)$, then it is the case that $e_{D}=f_{D}$.

Fix $x \in X$ and let $\lambda$ be an eigenvalue of $D_{x}$. Choose an enumeration of the spectrum of $D_{x}$ satisfying

$$
\begin{aligned}
& e_{D_{x}}(0)=\lambda \\
& e_{D_{x}}(1)=\lambda^{\prime}>\lambda .
\end{aligned}
$$

Find canonical neighborhoods $W=V \times(\lambda-\delta, \lambda+\delta), W^{\prime}=V \times\left(\lambda^{\prime}-\delta^{\prime}, \lambda^{\prime}+\delta^{\prime}\right)$ of $(x, \lambda)$ and $\left(x, \lambda^{\prime}\right)$ respectively.

Let $\mu_{0}(y)=\max \left\{\lambda \mid \lambda \in \operatorname{spec}\left(D_{y}\right)\right.$ and $\left.(y, \lambda) \in W\right\}$. Similarly, let $\mu_{1}(y)=$ $\min \left\{\lambda \mid \lambda \in \operatorname{spec}\left(D_{y}\right)\right.$ and $\left.(y, \lambda) \in W^{\prime}\right\}$.

Proposition 3.4. The functions $\mu_{0}$ and $\mu_{1}$ are continuous on $V$.

Proof. It will be sufficient to consider $\mu_{0}$, the case of $\mu_{1}$ being similar. Let $e_{0}(y) \leq \ldots \leq e_{k}(y)$ be the part of the spectrum of $D_{y}$ in $(\lambda-\delta, \lambda+\delta)$. Then $\mu_{0}(y)=e_{k}(y)$, and by the remark after Proposition 2.4, $\mu_{0}(y)$ is continuous.

Using $\mu_{0}$ and $\mu_{1}$ we define a spectral exhaustion over $V$ by taking, for each $y \in V$, the unique (not just up to translation) enumeration consistent with those choices. Thus, we have $\mu_{n}(y)$ defined for each integer $n$ and each $y \in V$.

Proposition 3.5. The functions $\mu_{n}$ are continuous on $V$ and, hence, $\left\{\mu_{n}\right\}$ is a spectral exhaustion over $V$. 
Proof. Choose a point $y \in V$. Taking a possibly smaller neighborhood $V^{\prime}$ of $y$, we get $n+1$ disjoint canonical neigborhoods of the form $V^{\prime} \times\left(\tilde{\mu}_{j}(y)-\delta_{j}, \tilde{\mu}_{j}(y)+\delta_{j}\right)$, where $\tilde{\mu}_{j}(y)$ are the eigenvalues of $D_{y}$ from $\mu_{0}(y)$ to $\mu_{n}(y)$ listed multiply. Now, for each $z \in V^{\prime}, \mu_{n}(z)$ is in the canonical neighborhood corresponding to the greatest real interval and it corresponds to one of the eigenvalues $\lambda_{r}(z)$ in it. We claim it must be the same $r$ for each $z$ in $V^{\prime}$. To see this, let $N$ be the number of eigenvalues in the canonical neighborhoods below the top one and let $r$ be the index corresponding to $\mu_{n}(y)$. Then $n=N+r$. If we look at a point $z$ and $\mu_{n}(z)=\lambda_{r^{\prime}}(z)$, then we still must have $n=N+r^{\prime}$, so that $r=r^{\prime}$. Thus, by Proposition 2.4, $\mu_{n}(z)$ varies continuously.

Doing this construction in a neighborhood of each point $x \in X$, we obtain a family of spectral exhaustions, each over an element of an open cover, $\left\{V_{i}\right\}$, where we may assume the open sets are connected. On the overlaps, any two exhaustions differ by an integer, so we obtain an integer valued 1-cochain relative to $\left\{V_{i}\right\}$ by taking the difference of the partial exaustions, $\nu_{i j}=\left.\mu_{0, i}\right|_{V_{i} \cap V_{j}}-\left.\mu_{0, j}\right|_{V_{i} \cap V_{j}}$ : $V_{i} \cap V_{j} \rightarrow \mathbb{Z}$. It is easily checked to be a cocycle and its cohomology class in $\check{H}^{1}(X, \mathbb{Z})$ will be defined to be the spectral flow of the family, $\operatorname{Sf}\left(\left\{D_{x}\right\}\right)$. It is straightforward to see that this definition agrees with other definitions of spectral flow. (c.f. [7]).

Theorem 3.6. A spectral exhaustion exists for the family $\left\{D_{x}\right\}$ if and only if the spectral flow of the family is zero, $\operatorname{Sf}\left(\left\{D_{x}\right\}\right)=0$.

Proof. If $\operatorname{Sf}\left(\left\{D_{x}\right\}\right)=0$ then the cocycle, which is defined with respect to the open cover $\left\{V_{i}\right\}$, is a coboundary, so that $\delta(\sigma)=\nu$ for some cochain $\sigma$. Then the 0 -cochain with components $\mu_{n, i}-\sigma_{n, i}$ can be used to define global functions $\mu_{n}$. These $\mu_{n}$ 's provide the required exhaustion.

For the converse, if an exhaustion exists, this determines the choices in constructing the cocycle representing $\operatorname{Sf}\left(\left\{D_{x}\right\}\right)$, and since the locally defined exhaustion functions all piece together to yield global functions, the class is equal to zero.

\section{Families with spectrum of constant multiplicity}

In this section we will obtain the first results relating spectral multiplicity to K-theory. Recall that we assume that the parameter space is a finite simplicial 
complex. While this assumption is not always necessary, the topology issues that would arise with additional generality are not fundamental ones.

Proposition 4.1. Suppose that the family $\left\{D_{x}\right\}$ has constant multiplicity at each point of a component, $\tilde{X}$, of $\Gamma$. Then $p r_{1}: \tilde{X} \rightarrow X$ is a covering.

Proof. We use Proposition 2.6 (ii) which states that, for each $x \in X$ and each eigenvalue $\lambda$ of $D_{x}$ there is a neighborhood, $V$ and a $\delta>0$ such that for each $y \in V, D_{y}$ has only one eigenvalue in the interval $[\lambda-\delta, \lambda+\delta]$. Then the function, $\sigma_{x, \lambda}: V \rightarrow \mathbb{R}$, which sends $y$ to that eigenvalue, is continuous.

It then follows that each component of $\Gamma\left(D_{x}\right)$ is a covering of $X$.

We defined the spectral flow of a family $\left\{D_{x}\right\}$ to be a 1-dimensional cohomology class,

$$
\operatorname{Sf}\left(\left\{D_{x}\right\}\right) \in \check{H}^{1}(X, \mathbb{Z}) .
$$

This class defines a homomorphism, for which we will use the same notation,

$$
\operatorname{Sf}\left(\left\{D_{x}\right\}\right): \pi_{1}(X) \rightarrow \mathbb{Z} .
$$

The following is an easy consequence of the definitions.

Proposition 4.2. If a component, $\tilde{X}$, of $\Gamma$ is a covering, $p r_{1}: \tilde{X} \rightarrow X$, then it corresponds to the homomorphism $\operatorname{Sf}\left(\left\{D_{x}\right\}\right): \pi_{1}(X) \rightarrow \mathbb{Z}$. i.e. image $\left(p r_{1 *}\right)=$ $\operatorname{ker}\left(\operatorname{Sf}\left(\left\{D_{x}\right\}\right)\right)$.

The next results give a criterion for the existence of a spectral exhaustion with disjoint graphs.

Theorem 4.3. Let $\left\{D_{x}\right\}$ be a family with spectrum of constant multiplicity; that $i s$, there exists an integer $k$ such that $m\left(D_{x}, \lambda\right)=k$, for each $(x, \lambda)$ in $\Gamma$. Assume that the spectral flow of the family is zero,

$$
\operatorname{Sf}\left(\left\{D_{x}\right\}\right)=0 .
$$

Then a spectral exhaustion with functions having disjoint images, (except for repeated functions due to multiplicity), exists.

Proof. Since the multiplicity of the covering is constant, each component is a covering. Moreover, each of these coverings corresponds to the homomorphism

$$
s f\left(\left\{D_{x}\right\}\right): \pi_{1}\left(X, x_{0}\right) \rightarrow \mathbb{Z},
$$


given by spectral flow. Thus, if the spectral flow of the family is zero, each of the coverings is a homeomorphism, so that $\Gamma \cong X \times \operatorname{spec}\left(D_{x_{0}}\right)$, for some point $x_{0} \in X$. Enumerate the spectrum of $D_{x_{0}}$ as $\left\{\lambda_{n}\left(x_{0}\right)\right\}$ and let $\tilde{X}_{n}$ be the component of $\Gamma$ containing $\lambda_{n}\left(x_{0}\right)$. Then set $\mu_{n}(x)=p r_{2} \circ\left(\left.p r_{1}\right|_{\tilde{X}_{n}}\right)^{-1}$. These functions satisfy the requirements to be a spectral exhaustion, and their graphs, being the components of $\Gamma$, are disjoint.

We obtain the following corollary from Remark 3.1.

Corollary 4.4. Let $\left\{D_{x}\right\}$ be a family with spectrum of constant multiplicity. Assume that the spectral flow of the family is zero,

$$
\operatorname{Sf}\left(\left\{D_{x}\right\}\right)=0 .
$$

Then the family $\left\{D_{x}\right\}$ is trivial in K-theory.

It is also worth noting the following result.

Corollary 4.5. Suppose that some component of $\Gamma$ is compact. Then the $K$ theory class of the family is trivial.

Proof. Let the component $\tilde{X}$ be compact. Then the number of sheets in the cover is the cardinality of $\pi_{1}(\tilde{X}) \cong \operatorname{image}\left(\operatorname{Sf}\left(\left\{D_{x}\right\}\right)\right)$, which must be finite. However, this is a subgroup of $\mathbb{Z}$, so it will have to be zero. Thus, the spectral flow of the family is zero and its class is trivial by Proposition 4.4.

Finally, we consider how the hypothesis of constant multiplicity can be replaced by an asymptotic version.

Theorem 4.6. Let $\left\{D_{x}\right\}$ be a family with $\operatorname{Sf}\left(\left\{D_{x}\right\}\right)=0$ Suppose that there exists an integer $N$ such that if $(x, \lambda) \in \Gamma$ and $\lambda>N$ then the family, $\left\{D_{x}\right\}$, has constant multiplicity at $(x, \lambda)$. Then the class of the family is trivial in $K^{1}(X)$.

Proof. Let $\Gamma_{R}=\{(x, \lambda): \lambda>R\}$. We will show that there is an $R>N$ so that some component of $\Gamma_{R}$ is a covering of $X$. If so, then as in $4.3, \operatorname{Sf}\left(\left\{D_{x}\right\}\right)=0$ will imply that this component is compact and by Corollary 4.5 the K-theory class of the family will be trivial.

Thus, we must show that there is a path component, $\tilde{X}$, of $\Gamma$ which is contained in $\Gamma_{R}$ for some $R>N$. Since $\operatorname{Sf}\left(\left\{D_{x}\right\}\right)=0$ a spectral exhaustion, $\mu_{n}$, exists. 
Let $\Gamma_{n}=$ image $\mu_{n}$. For each $x \in X$ there exists an $n_{x}$ and a neighborhood of $x$, $U_{x}$, such that $\mu_{n}(y)>N+1$ for all $y \in U_{x}$. Get a finite subcover, $U_{x_{1}}, \ldots, U_{x_{k}}$, and let $m=\max \left\{n_{x_{i}}\right\}$. Then $\mu_{m}(x)>N+1$ for all $x \in X$. This implies that the image of $\mu_{m}$ is a cover of $X$ and is compact and connected.

\section{FAmilies With BOUNDED MULTiPLiCity}

In this section we will consider the question of when an element of odd Ktheory can be represented by a family with uniform bounded multiplicity. To this end let, for $n \geq 1, \mathcal{F}_{R}^{s a}(n)$ denote the operators with multiplicity less than or equal to $n$. Then $\mathcal{F}_{R}^{s a}(n) \subseteq \mathcal{F}_{R}^{s a}(n+1)$ and we set $\mathcal{F}_{R}^{s a}(\infty)=\bigcup \mathcal{F}_{R}^{s a}(n)$. We do the same for $\mathcal{F}_{R}$. Throughout, $X$ will be a compact space.

Recall that in Atiyah-Singer, [4], the following diagram was studied.

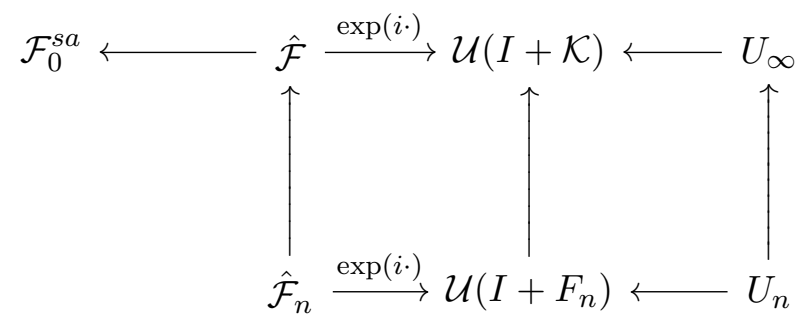

Here, $\mathcal{F}_{0}^{s a}$ is the bounded self-adjoint Fredholms with essential spectrum on both sides of the origin, while $\hat{\mathcal{F}}$ is those operators with norm 1 and essential spectrum \pm 1 . Also, $\mathcal{U}\left(I+F_{n}\right)$ is the unitary operators of the form $I+K$, with $K$ of rank $n$, $\hat{\mathcal{F}}_{n}$ is the operators in $\hat{\mathcal{F}}$ with finitely many eigenvalues in $(-1,1)$ and for which $\exp (i T) \in U_{n}$. The unlabeled arrows are inclusions. The Atiyah-Singer result shows that the composition of the maps on the top row and their appropriate homotopy inverses provide a homotopy equivalence which we shall denote by $\hat{\chi}: \mathcal{F}_{0}^{s a} \rightarrow U_{\infty}$. There is an obvious inclusion map of $\mathcal{F}_{R}^{s a}$ into $\mathcal{F}_{0}^{s a}$ and hence into $U_{\infty}$.

To study the question of bounded multiplicity we make the following definition.

Definition 5.1. Let $K_{(\infty)}^{1}(X)$ be the subset of $K^{1}(X)$ consisting of classes $[\alpha]$, $\alpha: X \rightarrow \mathcal{F}_{R}^{s a}$, such that there is an $n$ and an $\alpha^{\prime} \simeq \alpha$ with $\alpha^{\prime}: X \rightarrow \mathcal{F}_{R}^{\text {sa }}(n)$. Let $K_{(\infty)}^{0}(X)$ be defined in an analogous way using $\mathcal{F}^{R}$.

Note that the homotopy between $\alpha$ and $\alpha^{\prime}$ is allowed to run through all of $\mathcal{F}_{0}^{s a}$. 
Proposition 5.2. $K_{(\infty)}^{*}(X)$ is a natural subgroup of $K^{*}(X)$.

Proof. This subgroup is clearly preserved by induced homomorphisms and contains the identity element of $K^{*}(X)$. Addition in $K^{1}(X)$ is induced by composition of operators which is homotopic to orthogonal direct sum. Thus, the sum of classes represented by bounded multiplicity elements is also represented by a family of bounded multiplicity. Moreover, since the inverse of an element given by a family $\alpha: X \rightarrow \mathcal{F}_{0}^{s a}$ is represented by $-\alpha$, this operation preserves the property of having bounded multiplicity. Thus, the result follows.

Proposition 5.3. $K_{(\infty)}^{*}(X)$ is mapped to itself under Bott periodicity.

Proof. The Bott periodicity map is given by taking the product with the Bott element of $\tilde{K}^{0}\left(S^{2}\right)$. The product operation can be realized in the present context by letting each operator in the family act on the Hilbert space obtained by tensoring with the $L^{2}$ sections of the bundles representing the $K^{0}$ class. If the bundle is trivial, then the multiplicity will by multiplied by its dimension. If it is not trivial, then it is a summand of a trivial bundle and one can see that restricting to the image of the projection onto the sections of the bundle can only lower the multiplicity. Note that in this setting, the operators in the family will commute with the projections onto the sections of the bundle.

We will now make use of a construction which appears in a paper of Mickelsson, [12]. It will be used to associate to a map into the finite dimensional unitary group, $U_{n}$, an explicit family of unbounded self-adjoint Fredholm operators with the multiplicity of their spectrum uniformly bounded by $n$.

Let $U \in U_{n}$. Consider the operator

$$
-i \frac{d}{d x}: C^{\infty}\left([0,1], \mathbb{C}^{n}\right) \rightarrow C^{\infty}\left([0,1], \mathbb{C}^{n}\right)
$$

with the boundary condition

$$
\xi(1)=U \xi(0) .
$$

This yields a self-adjoint Fredholm operator on $L^{2}\left([0,1], \mathbb{C}^{n}\right)$ which we will denote $D_{U}$. It is straightforward to compute the spectrum of $D_{U}$ and the result is as follows. Let $\left\{z_{1}, \ldots, z_{n}\right\}$ be the spectrum of $U$. Let $\lambda_{j}$ satisfy $0 \leq \lambda_{j}<1$ and $z_{j}=e^{2 \pi i \lambda_{j}}$. Then,

$$
\operatorname{spec}\left(D_{U}\right)=\left\{m+\lambda_{j} \mid m \in \mathbb{Z}, 1 \leq j \leq n\right\} .
$$


The multiplicity of the eigenvalue $m+\lambda_{j}$ is the same as that of the eigenvalue $z_{j}$ of $U$, and it follows that the multiplicity of the spectrum of $D_{U}$ is less than or equal to $n$.

Let $\mu_{n}: U_{n} \rightarrow \mathcal{F}_{R}^{s a}$ be defined by $\mu_{n}(U)=D_{U}$. We will refer to $\mu_{n}$ as the Mickelsson map.

Proposition 5.4. The Mickelsson map yields a map

$$
\mu: U_{\infty}=\bigcup_{n \geq 1} U_{n} \rightarrow \mathcal{F}_{R}^{s a} \rightarrow \mathcal{F}_{0}^{s a},
$$

which induces an isomorphism on homotopy groups,

$$
\mu_{*}: \pi_{i}\left(U_{\infty}\right) \rightarrow \pi_{i}\left(\mathcal{F}_{0}^{s a}\right),
$$

for all $i$.

Proof. The first statement follows from the definitions while the second is a consequence of the facts that the Mickelsson map commutes with periodicity and the computation from [12] that it is an isomorphism for $S^{3}$.

Note that if $X$ is a compact space, then $\mu_{*}:\left[X, U_{\infty}\right] \rightarrow\left[X, \mathcal{F}_{0}^{s a}\right]$ actually maps into $K_{\infty}^{1}(X)$.

These three propositions yield the following theorem.

Theorem 5.5. Let $X$ be a compact metric space. Then one has

$$
K_{(\infty)}^{*}(X)=K^{*}(X)
$$

Proof. It follows from Propositions 5.2 and 5.3 that $K_{(\infty)}^{*}(X)$ defines a cohomology theory on compact spaces with a 6 -term exact sequence of the same type as that for $K^{*}(X)$. The inclusion induces a map of 6-term sequences. Assume first that $X$ is a finite complex. Then applying the cohomology theories to the sequence of skeletons,

$$
X^{(k)} \rightarrow X^{(k+1)} \rightarrow \bigvee S^{(k+1)}
$$


will yield the result by induction once one knows that it holds for spheres. However, for spheres the Mickelsson map composed with the inclusion,

$$
\pi_{i}\left(U_{\infty}\right) \stackrel{\mu_{*}}{\longrightarrow} \pi_{i}\left(\mathcal{F}_{0}^{s a}(\infty)\right) \stackrel{i}{\longrightarrow} \pi_{i}\left(\mathcal{F}_{0}^{s a}\right)
$$

agrees with the isomorphism from Atiyah-Singer, [4]. Here, $\mathcal{F}_{0}^{s a}(\infty)$ denotes the subset of $\mathcal{F}_{0}^{s a}$ homotopic to regular operators of bounded multiplicity. By Proposition 5.4, $\mu_{*}$ is an isomorphism on spheres, hence so is the inclusion, $i$. This proves the result for finite complexes. By expressing a compact metric space as an inverse limit of finite complexes one obtains the desired conclusion.

Note that the same argument shows that the Mickelsson map is an isomorphism.

This result has connections to the paper of Nicolaescu, [13], in which the relation of $K^{1}(X)$ and homotopy classes of maps into the space of unbounded selfadjoint Fredholm operators with essential spectrum $\{ \pm 1\}$, but possibly having some continuous spectrum, is addressed. From the vantage of this paper Theorem 5.5 shows that every element in $K^{1}(X)$ is represented by a family of regular unbounded self-adjoint Fredholm operators.

As a consequence of this fact, one sees that any family is homotopic to a family with bounded multiplicity. One can estimate the bound on the multiplicity in a rough way using the dimension of $X$. It would be desirable to get a refined estimate based on the topology of $X$.

Definition 5.6. Let $\left\{D_{x}\right\}$ be a family on $X$. The minimal multiplicity of the family is the least integer $n$ such that $\left\{D_{x}\right\} \cong\left\{D_{x}\right\}^{\prime}$ where $\left\{D_{x}\right\}^{\prime}$ is a family with multiplicity bounded by $n$.

Proposition 5.7. Let $[\alpha] \in K^{1}(X)$ and suppose the dimension of $X$ is $k$. Then $[\alpha]$ is represented by a family $\left\{D_{x}\right\}$ with minimal multiplicity $<\left[\frac{k+1}{2}\right]$, where $[x]$ denotes the largest integer less than or equal to $x$.

Proof. Suppose that $\alpha: X \rightarrow U_{N}$ is given. Using the fibrations $U_{n-1} \rightarrow U_{n} \rightarrow$ $S^{2 n-1}$ one can inductively reduce the dimension of the unitary group to the least possible, which is $\left[\frac{k+1}{2}\right]$. The result follows upon applying Theorem 5.5. 


\section{Multiplicity $\leq 2$}

As a sample of how conditions on the multiplicity beyond assuming constancy can be used to study the K-theory class of a family, we will consider the case when the multiplicity is less than or equal to 2 . We will also assume that the spectral flow of the family is zero. The main result of this section is that, if we assume that the space $X$ has no torsion in cohomology, then such a family is trivial in K-theory if a certain 3-dimensional cohomology class vanishes. Thus, the index gerbe will be zero also.

Let us assume that we have a family with $\operatorname{mult}\left(\left\{D_{x}\right\}\right) \leq 2$ and recall the standing assumption that the parameter space $X$ is a connected finite simplicial complex. Assume $\operatorname{Sf}\left(\left\{D_{x}\right\}\right)=0$ and let $\left\{\mu_{n}\right\}$ be an exhaustion. Our procedure will be to deform the family inductively over k-skeletons for increasing $k$, so that the exhaustion for the deformed family, $\left\{\tilde{\mu}_{n}\right\}$, has $\tilde{\mu}_{0}(x)<\tilde{\mu}_{1}(x)$ for all $x$. The triviality will then follow from Proposition 2.8.

Let $C_{i, i+1}=\left\{x \mid \mu_{i}(x)=\mu_{i+1}(x)\right\}$, for any $i \in \mathbb{Z}$. Since $\operatorname{mult}\left(\left\{D_{x}\right\}\right) \leq 2$ we have $C_{-1,0}, C_{0,1}$ and $C_{1,2}$ disjoint closed sets. Let $W_{i, i+1}, i=-1,0,1$, be disjoint open neighborhoods of $C_{i, i+1}$. We assume the triangulation of $X$ so fine that any closed simplex which meets $C_{i, i+1}$ is contained in $W_{i, i+1}$. Thus, there are a finite number of simplices, $\sigma_{l}$, such that

$$
C_{i, i+1} \subseteq \operatorname{interior}\left(\bigcup_{1}^{n} \sigma_{l}\right) \subseteq \bigcup_{1}^{n} \sigma_{l} \subseteq W_{i, i+1} .
$$

Our procedure for deforming a family involves successive application of certain types of "moves". The first is a preliminary flattening process which allows one to control the geometry of the sets over which the family has eigenvalues of multiplicity 2. We will state things for $C_{0,1}$ to simplify notation, but all results hold for $C_{i, i+1}$ with the appropriate modifications.

Proposition 6.1 (Flattening). Let $K$ be a closed subset of $C_{0,1}$ and let $W_{1}, W_{2}$ be open sets with compact closures satisfying $K \subseteq W_{1} \subseteq \bar{W}_{1} \subseteq W_{2} \subseteq \bar{W}_{2} \subseteq W_{0,1}$. Assume further that $K=C_{0,1} \cap W_{2}$. Then there exists a family $\left\{\tilde{D}_{x}\right\}$ with associated exhaustion $\left\{\tilde{\mu}_{n}\right\}$, which satisfies

i) $K \subseteq W_{2} \cap \tilde{C}_{0,1}=\bar{W}_{1}$, where $\tilde{C}_{0,1}=\left\{x \mid \tilde{\mu}_{0}(x)=\tilde{\mu}_{1}(x)\right\}$,

ii) $\tilde{D}_{x}=D_{x}$ for $x \in X \backslash W_{2}$, and 
iii) $\left\{\tilde{D}_{x}\right\} \simeq\left\{D_{x}\right\}$.

Proof. Let $\phi: X \rightarrow[0,1]$ be a function satisfying

$$
\phi(x)= \begin{cases}0 & \text { for } x \in X \backslash W_{2} \\ 1 & \text { for } x \in \bar{W}_{1}\end{cases}
$$

Define

$$
\tilde{D}_{x, t}=D_{x}+t \phi(x)\left(h_{x}\left(D_{x}\right)-D_{x},\right.
$$

where $h_{x}: \mathbb{R} \rightarrow \mathbb{C}$ is a continuous function satisfying

$$
h_{x}(t)=\left\{\begin{array}{ll}
t & \text { for } t \leq \mu_{-1}(x) \text { or } t \geq \mu_{1}(x) \\
\mu_{1}(x) & \text { for } \mu_{0}(x) \leq t \leq \mu_{1}(x) \\
\lambda_{x}(t) & \text { for } \mu_{-1}(x) \leq t \leq \mu_{0}(x)
\end{array},\right.
$$

where $\lambda_{x}(t)$ is the linear function with graph connecting $\left(\mu_{-1}(x), \mu_{-1}(x)\right)$ to $\left(\mu_{0}(x), \mu_{1}(x)\right)$

Letting $\tilde{D}_{x}=\tilde{D}_{x, 1}$, with associated exhaustion $\tilde{\mu}_{n}(x)$, one checks that $K \subseteq$ $W_{2} \cap \tilde{C}_{0,1}=\bar{W}_{1}$ and that the conclusions of the proposition hold for the family $\left\{\tilde{D}_{x}\right\}$.

Thus, the preceeding deformation allows one to determine the set precisely, $\left(\bar{W}_{1}\right.$ above), on which multiplicity of $\left(x, \tilde{\mu}_{0}(x)\right)$ is 2 . Next, we will modify the family over neighborhoods of these sets.

Let $X^{(0)}$ be the 0 -skeleton of the parameter space $X$. The first step will be to deform the family $\left\{D_{x}\right\}$ on a neighborhood of $X^{(0)}$.

Proposition 6.2. Let $y \in X^{(0)}$. Then there exists a contractible neighborhood $V$ with $V \cap X^{(0)}=\{y\}$ and a family $\left\{\tilde{D}_{x}\right\}$ satisfying

i) $\tilde{D}_{x}=D_{x}$ for $x \in X \backslash V$,

ii) $\left\{\tilde{D}_{x}\right\} \simeq\left\{D_{x}\right\}$, and

iii) There is a neighborhood $W$ of $y$ such that $\tilde{\mu}_{0}(x)<\tilde{\mu}_{1}(x)$ for $x \in W \subseteq$ $\bar{W} \subseteq V$.

Proof. If $\mu_{0}(y)<\mu_{1}(y)$ then this uniquely will hold in a neighborhood of $y$ and the original family will satisfy conditions (i)-(iii). If, on the other hand, $y \in C_{0,1}$, so that $\mu_{-1}(y)<\mu_{0}(y)=\mu_{1}(y)<\mu_{2}(y)$, then we apply Proposition 6.1 to obtain 
a contractible neigborhood with compact closure, $V$, of $y$ on which $\mu_{0}(x)=\mu_{1}(x)$ for $x \in V$.

Let $\mathcal{E}=\left\{(x, v) \in V \times \mathcal{H} \mid v\right.$ is in the span of the eigenvectors for $\mu_{0}(x)$ and $\left.\mu_{1}(x)\right\}$. Then $\mathcal{E} \rightarrow X$ is a 2 -dimensional vector bundle on some, possibly smaller, neighborhood of $y$ which we continue to call $V$. Since $V$ is contractible, the bundle is trivial. Thus, there exists a framing $\left\{\sigma_{0}, \sigma_{1}\right\}$, where $\sigma_{j}(x)$ is an eigenvector for $\mu_{j}(x)$, for $j=0,1$. Shrink $V$ to get $W \subseteq \bar{W} \subseteq V$ and, using a bump function $\phi$, we extend $\sigma_{i}$ to all of $X$. Let $\alpha(t, x)=t \frac{\mu_{1}(x)+\mu_{2}(x)}{2}$, and set $\tilde{D}_{x, t}=D_{x}+\alpha(t, x) P_{\sigma_{1}(x)}$, where $P_{\sigma_{1}(x)}$ is the orthogonal projection onto the subspace spanned by $\sigma_{1}(x)$. The family $\left\{\tilde{D}_{x, 1}\right\}$ satisfies the requirements of the proposition.

We repeat this construction, with the obvious modifications, for vertices in $C_{-1,0}$. Alternatively, one may observe that it is possible to apply this method to the vertices in both $C_{-1,0}$ and $C_{0,1}$ simultaneously.

Thus, we have deformed our family so that $\mu_{0}(x)<\mu_{1}(x)$ on a neighborhood of the 0 -skeleton. We will now proceed inductively to extend this separation to all of $X$.

It is worth noting that the previous deformations and all future ones have the property that they are monotone, in the sense that $\mu_{i}(x) \leq \tilde{\mu}_{i}(x)$ for all $i$ and $x \in X$.

From now on, to simplify notation, we will drop the tilde and rename the family resulting from a deformation as $\left\{D_{x}\right\}$.

Assume that our family has the property that $\mu_{0}(x)<\mu_{1}(x)$ on a neighborhood, $W$, of the (k-1)-skeleton. Let $\mathbb{C}^{2} \rightarrow \mathcal{E} \rightarrow W$ be the 2 -dimensional vector bundle whose fiber over the point $x$ is the span of the eigenspaces for $\mu_{0}(x)$ and $\mu_{1}(x)$. For $x \in W$, define $\sigma_{0}(x)$ to be the orthogonal projection onto the eigenspace for $\mu_{0}(x)$. This defines a continuous field of projections over $W$. The next result shows that one can obtain the desired deformation of $\left\{D_{x}\right\}$ if the field $\sigma_{0}$ can be extended to the k-skeleton. We will construct the deformation simplex by simplex.

Proposition 6.3. Let $\left\{D_{x}\right\}$ be a family of operators with $\mu_{0}(x)<\mu_{1}(x)$ on a neighborhood, $W$, of the (k-1)-skeleton. Let $\Delta^{k}$ be a k-simplex. Let ${ }^{\prime} \Delta^{k}$ be a smaller k-simplex with $C_{0,1} \cap \Delta^{k} \subseteq$ interior $\left({ }^{\prime} \Delta^{k}\right) \subseteq{ }^{\prime} \Delta^{k} \subseteq \operatorname{interior}\left(\Delta^{k}\right)$. If $\left.\sigma_{0}\right|_{\partial\left({ }^{\prime} \Delta^{k}\right)}$ extends to a field of projections onto the eigenspaces for $\mu_{0}(x)$ over $\Delta^{k}$, 
then there are neighborhoods, $V$ and $W^{\prime}$, with $X^{(k-1)} \cup \Delta^{k} \subseteq W^{\prime} \subseteq \bar{W}^{\prime} \subseteq V$ and a family $\left\{\tilde{D}_{x}\right\}$ satisfying

i) $\tilde{D}_{x}=D_{x}$ for $x \in X \backslash V$,

ii) $\left\{\tilde{D}_{x}\right\} \simeq\left\{D_{x}\right\}$, and

iii) $\tilde{\mu}_{0}(x)<\tilde{\mu}_{1}(x)$ for $x \in W^{\prime}$.

Proof. Let $\phi(x)$ be a bump function which is 1 on $W^{\prime}$ and 0 on $X \backslash V$. Let $\tilde{D}_{x, t}=D_{x}+t \phi(x)\left(\frac{\mu_{1}(x)+\mu_{2}(x)}{2}\right) \sigma_{0}(x)$. Then it is straightforward to check that the family $D_{x, 1}$ satisfies conditions (i) - (iii).

In order to use this construction to deform a family which is separated over a neighborhood of $X^{(k-1)}$, we apply Proposition 6.1. For this, note that $C_{0,1}$ is contained in the interior of $\mathrm{k}$-simplices. Consider one such simplex and find a subsimplex with parallel sides which contains its intersection with $C_{0,1}$ in its interior. We may assume that the boundary of the smaller simplex is contained in the open set over which $\mu_{0}(x)<\mu_{1}(x)$. Next one applies the flattening lemma to obtain a new family which has the smaller simplex as exactly $C_{0,1} \cap \Delta^{k}$. The projection field is defined on $\partial\left({ }^{\prime} \Delta^{k}\right)$. Thus, if one can alway extend these projection fields from the boundary of a k-simplex to the interior, then we can accomplish the deformation of the family to one for which $\mu_{0}(x)<\mu_{1}(x)$ over a neighborhood of the k-skeleton.

Note that, since $\Delta^{k}$ is contractible, the bundle $\mathcal{E} \rightarrow X$ is trivial over $\Delta^{k}$. Thus, the existence of the extension is equivalent to the map $\sigma_{0}: \partial \Delta^{k} \rightarrow G r_{2}\left(\mathbb{C}^{2}\right)$ being null-homotopic. Here $G r_{2}\left(\mathbb{C}^{2}\right)$ is the Grassmannian of lines in $\mathbb{C}^{2}$, which is homeomorphic to $S^{2}$. Since $\pi_{0}\left(S^{2}\right)=\pi_{1}\left(S^{2}\right)=0$, one can always obtain a deformed family for which $\mu_{0}(x)<\mu_{1}(x)$ on a neighborhood of the 2-skeleton, $X^{(2)}$. However, since $\pi_{2}\left(S^{2}\right)=\mathbb{Z}$, it is not clear that one can proceed. We shall address this in the application below.

Putting these facts together we briefly present a sample of the type of result obtainable using these methods. Note that the result below makes the case that the multiplicity of eigenvalues has a strong effect on the classification of families of operators.

Theorem 6.4. Let $\left\{D_{x}\right\}$ be a family on $S^{2 n+1}$ with $n \geq 2$. If the multiplicity is less than or equal to 2, then the family is rationally trivial in K-theory. 
Proof. We sketch the argument. Note that given a separation over an open set, we get a continuous eigenprojection field over that set. Thus, as above, we may obtain an eigenprojection field over a neighborhood of the 2-skeleton. For the induction step, let $k<2 n+1$ and assume that in a neighborhood of the $k$-skeleton we have $\mu_{-1}(x)<\mu_{0}(x)<\mu_{1}(x)$. Thus there is a rank 1 eigenprojection field over this neighborhood. We will deform the family so that this property holds over the $k$-skeleton, hence on a neighborhood of it.

We now try to extend the eigenprojection field over the 3 -skeleton. Proceeding as above by flattening and deforming, we obtain an eigenprojection field over the boundaries of 3-simplices. We take a slightly different approach to complete the deformation process. We first try to extend to simply a general projection field. This can be done if the eigenprojection fields defined on the boundaries of the 3 -simplices, $S^{2} \rightarrow G r_{1}(\mathcal{H})$, are null-homotopic. Since $G r_{1}(\mathcal{H})=\mathbb{C} P^{\infty}$, this is not automatic. However, obstruction theory applies and there is a class in $H^{3}\left(S^{2 n+1}, \pi_{2}\left(G r_{1}(\mathcal{H})\right)\right.$ which must vanish in order for the extension to exist (after going back and redefining over lower skeleta). Since we are working with a sphere of dimension greater than 5 , this group vanishes and the extension exists.

We now proceed by induction. We have a field over all of $S^{2 n+1}$ which is an eigenprojection field over a neighborhood of the $k$-skeleton. We next try to push this field down to be an eigenprojection field over the $(k+1)$-skeleton. We consider the set of $(k+1)$-simplices and their boundaries. There are two cases. If a $(k+1)$-simplex doesn't contain any singular points, then we have two projection fields over it-an eigenprojection field and the general one we just obtained. Any two are homotopic relative to the boundary because only the second homotopy group of $G r_{1}(\mathcal{H})$ is non-zero. We will assign 0 to such a simplex. For the others, using the flattening procedure described above, we will get an element in the relative homotopy group, $\pi_{k}\left(G r_{1}(\mathcal{H}), G r_{1}\left(\mathbb{C}^{2}\right)\right) \cong \pi_{k-1}\left(G r_{1}\left(\mathbb{C}^{2}\right)\right)=\pi_{k-1}\left(S^{2}\right)$. This will define an obstruction class in $H^{k+1}\left(S^{2 n+1},\left(S^{2 n+1}\right)^{(k)} ; \pi_{k-1}\left(S^{2}\right)\right)$.

We note that, for any complex $X$, one has $H^{j}\left(X, X^{(k)}\right)=0$ if $j \leq k$, and $H^{j}\left(X, X^{(k)}\right) \cong H^{j}(X)$ if $j>k$. Thus, the only group which can be non-zero is $\left.H^{2 n+1}\left(S^{2 n+1},\left(S^{2 n+1}\right)^{(2 n)}\right) ; \pi_{2 n-1}\left(S^{2}\right)\right)$, a torsion group. For $k<2 n+1$ we extend the restriction of the eigenprojection field to the $(k-1)$-skeleton to the $(k+1)$-skeleton and use this to deform the family so it is separated there. For the case of the top dimension the following procedure takes care of this obstacle. 
We have a possibly non-trivial, obstruction class which would belong to $H^{2 n+1}$ $\left(S^{2 n+1} ; \pi_{2 n}\left(G r_{1}(\mathcal{H}), G r_{1}\left(\mathbb{C}^{2}\right)\right)\right)$. Since $n \geq 2, \pi_{2 n}\left(G r_{1}(\mathcal{H}), G r_{1}\left(\mathbb{C}^{2}\right)\right) \cong \pi_{2 n-1}\left(S^{2}\right)$ is a finite group, say of order $N$. We will show that $N\left\{D_{x}\right\}$ is trivial, and hence $\left\{D_{x}\right\}$ is rationally trivial. Let $\Delta$ be a $2 n+1$-simplex which meets $C_{0,1}$ in its interior and let $\Delta^{\prime}$ be a sub-simplex obtained by flattening. Thus, we may assume that the family has multiplicity two at each point of $\Delta^{\prime}$ and there is a rank 1 eigenprojection field along the boundary. Now, consider the family $N\left\{D_{x}\right\}$. We trivialize each 2-dimensional eigenbundle separately and get a map

$$
c(\Delta):\left(\Delta^{\prime}, \partial \Delta^{\prime}\right) \rightarrow\left(G r_{1}(\mathcal{H}) \times \ldots \times G r_{1}(\mathcal{H}), G r_{1}\left(\mathbb{C}^{2}\right) \times \ldots \times G r_{1}\left(\mathbb{C}^{2}\right)\right)
$$

defining a class in $\pi_{2 n}\left(G r_{1}(\mathcal{H}), G r_{1}\left(\mathbb{C}^{2}\right)\right) \oplus \ldots \oplus \pi_{2 n}\left(G r_{1}(\mathcal{H}), G r_{1}\left(\mathbb{C}^{2}\right)\right)$. The map induced by addition on homotopy groups sends the class of this map to zero in $\pi_{2 n}\left(G r_{1}(\mathcal{H}), G r_{1}\left(\mathbb{C}^{2}\right)\right)$. From the commutative diagram,

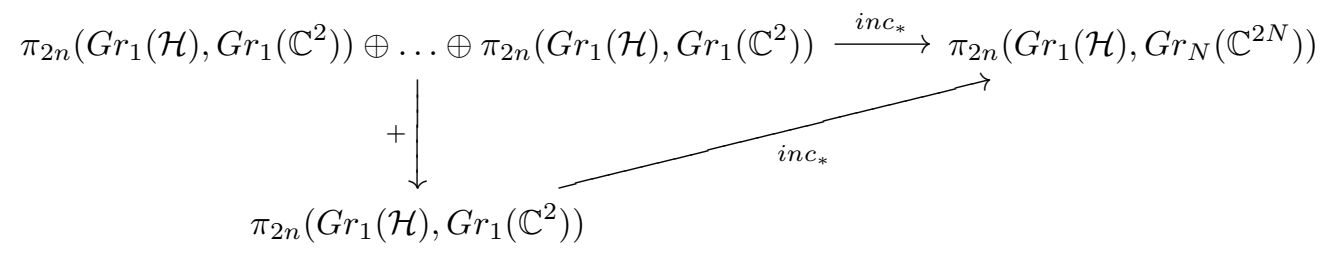

we see that we may deform the map $c(\Delta): \Delta^{\prime} \rightarrow G r_{1}(\mathcal{H})$ to one mapping into $G r_{N}\left(\mathbb{C}^{2 N}\right)$. Using the same deformation as in the rank 1 case, we obtain a new family for which $\mu_{0}(x)<\mu_{1}(x)$ for all $x \in \Delta^{\prime}$. Doing this process over each $n$-simplex yields the required trivial family.

\section{CONCluding REMARKS}

The intention of the present paper was to begin a study of the manner in which the variation of the eigenvalues and eigenspaces of a family of self-adjoint Fredholm operators effects the K-theory class of the family. While we showed that the behavior of the multiplicity function can effect the topology of the family, there is much yet to be resolved. Some questions which seem essential to making further progress are listed below.

- How is the 3-dimensional integral cohomology class which arises when applying obstruction theory related to the index gerbe, c.f. Lott, [10]. If they determine each other, can one obtain all the components of the Chern character of the family using these methods? 
- Suppose $[\alpha] \in K^{1}(X)$ and there is an $\alpha^{\prime}$ with $\alpha \simeq \alpha^{\prime}$ and with the multiplicity of $\alpha^{\prime}$ bounded by $n$. Let $\mathcal{M}([\alpha])$ be the least such $n$. If $[\alpha] \neq 0$ then $\mathcal{M}([\alpha])>1$. How are the topological invariants of $[\alpha]$ related to $\mathcal{M}([\alpha])$ ?

- The equivalence relation generated by the "moves" we are using to deform the families is possibly stronger than homotopy. Is one obtaining a more refined type of K-theory in this way?

- It would be interesting to know in what sense the K-theory class of a family is determined by a finite part of the spectrum. To be more precise, suppose $\left\{D_{x}\right\}$ is a family with multiplicity bounded by $n$. Is there an integer $\mathcal{N}(n)$ so that the part of the graph of the family, $\bigcup_{|k|<\mathcal{N}(n)} \mu_{k}(X)$, along with the corresponding eigenspaces, determines whether the family is trivial (or rationally trivial) in K-theory?

- Although various partial results similar to those in the last section are known to the authors, the appropriate general statement has not yet been obtained. We expect that the following will hold. Assume the parameter space of the family, $X$, is an n-dimensional finite complex and the spectral flow of the family is zero. Further, suppose there is an element of the exhaustion, $\mu_{k}$ such that the multiplicity at any point of $\mu_{k}(X)$ is $N$ or $N+1$, where $N>n$. Then, if the 3-dimensional obstruction obtained above is zero, the family is rationally trivial in K-theory.

\section{REFERENCES}

[1] M. F. Atiyah, K-theory, Benjamin Press, New York, 1967.

[2] M. F. Atiyah and F. Hirzebruch, Vector bundles and homogeneous spaces, Proc. Sympos. Pure Math., Vol. III, American Mathematical Society, Providence, R.I., 1961, pp. 7-38.

[3] M. F. Atiyah and I. M. Singer, The index of elliptic operators I, Annals of Mathematics 87 (1968), 484-530.

[4] M. F. Atiyah and I. M. Singer, Index theory for skew-adjoint Fredholm operators, Inst. Hautes Études Sci. Publ. Math. (1969), no. 37, 5-26. MR MR0285033 (44 \#2257)

[5] Dirac operators coupled to vector potentials, Proc. Nat. Acad. Sci. U.S.A. 81 (1984), no. 8, Phys. Sci., 2597-2600. MR MR742394 (86g:58127)

[6] M. F. Atiyah, I. M. Singer, and V. K. Patodi, Spectral asymmetry and Riemannian geometry, I, Math. Proc. Camb. Phil. Soc. 77 (1975), 43-69.

[7] B. Boos-Bavnbek and K. P. Wojciechowski, Elliptic boundary problems for Dirac operators, Birkhäuser, 1993. 
[8] Klaus Jänich, Vektorraumbündel und der Raum der Fredholm-Operatoren, Math. Ann. 161 (1965), 129-142.

[9] Michael Joachim, Unbounded Fredholm operators and K-theory, High-dimensional manifold topology, World Sci. Publ., River Edge, NJ, 2003, pp. 177-199. MR MR2048722 (2005c:46104)

[10] John Lott, Higher-degree analogs of the determinant line bundle, Comm. Math. Phys. 230 (2002), no. 1, 41-69. MR MR1930571 (2003j:58052)

[11] Richard B. Melrose and Paolo Piazza, Families of Dirac operators, boundaries and the b-calculus, J. Differential Geom. 46 (1997), no. 1, 99-180. MR MR1472895 (99a:58144)

[12] J. Mickelsson, Gerbes and quantum field theory, arXiv: math-ph/060303.

[13] L. Nicolaescu, On the space of Fredholm self-adjoint operators, An. Sti. Univ. Iasi 53 (2007), 209-227.

[14] I. M. Singer, Families of Dirac operators with applications to physics, Astérisque (1985), no. Numero Hors Serie, 323-340, The mathematical heritage of Élie Cartan (Lyon, 1984).

Ronald G. Douglas

Department of Mathematics

Texas A\&M University

College Station, TX 77843-3368

E-mail: rdouglas@math.tamu.edu

Jerome Kaminker

Department of Mathematics

UC Davis

Davis, CA 95616

E-mail: kaminker@math.ucdavis.edu 